\title{
Dressings as an adjunct to pressure ulcer prevention: consensus panel recommendations
}

\author{
Joyce Black ${ }^{1}$, Michael Clark ${ }^{2}$, Carol Dealey ${ }^{3}$, Christopher T Brindle $^{4}$, Paulo Alves ${ }^{5}$, Nick Santamaria ${ }^{6}$ \& \\ Evan Call ${ }^{7}$
}

1 Adult Health and Illness Department, College of Nursing, University of Nebraska Medical Center, Omaha, NE, USA

2 Birmingham City University, Birmingham, UK

3 Birmingham NHSFT, University of Birmingham and University Hospital, Birmingham, UK

4 Wound Care Team, Virginia Commonwealth University (VCU) Medical Center, Richmond, VA, USA

5 Institute of Health Sciences, Catholic University of Portugal, Porto, Portugal

6 Translational Research, University of Melbourne \& Royal Melbourne Hospital AU, Melbourne, Australia

7 Department of Microbiology, Weber State University, Salt Lake City, UT, USA

\section{Key words}

Pressure ulcer prevention; Preventive dressings; ICU pressure ulcers

\section{Correspondence to}

J Black

Associate Professor of Nursing

University of Nebraska Medical Center

Omaha

NE, USA

E-mail: jblack@unmc.edu
Black J, Clark M, Dealey C, Brindle CT, Alves P, Santamaria N, Call E. Dressings as an adjunct to pressure ulcer prevention: consensus panel recommendations. Int Wound J 2014; doi: 10.1111/iwj.12197

\section{Abstract}

The formulation of recommendations on the use of wound dressings in pressure ulcer prevention was undertaken by a group of experts in pressure ulcer prevention and treatment from Australia, Portugal, UK and USA. After review of literature, they concluded that there is adequate evidence to recommend the use of five-layer silicone bordered dressings (Mepilex Border Sacrum ${ }^{\circledR}$ and 3 layer Mepilex Heel ${ }^{\circledR}$ dressings by Mölnlycke Health Care, Gothenburg, Sweden) for pressure ulcer prevention in the sacrum, buttocks and heels in high-risk patients, those in Emergency Department (ED), intensive care unit (ICU) and operating room (OR). Literature on which this recommendation is based includes one prospective randomised control trial, three cohort studies and two case series. Recommendations for dressing use in patients at high risk for pressure injury and shear injury were also provided.
Pressure ulcer prevention has been aided by clinical practice guidelines since 1994 (7). However, in the last 20 years little progress has been made or sustained in preventing pressure ulcers in the high-risk patient in critical care units or the operating room (OR). The National Pressure Ulcer Advisory Panel (NPUAP) 2012 monograph examined the trend in pressure ulcers over the previous decade (8). Their work reported that the incidence of pressure ulcers in critical care ranges between $3.3 \%$ and $53.4 \%$ and prevalence rates for critically ill patients ranged between $25.1 \%$ and $45.5 \%$ (9). In the ORs, the incidence of pressure ulcers ranged from $5 \%$ to $53.4 \%$ (10). In the most recent guideline in 2009 , the use of dressings for prevention of pressure ulcers was not addressed, apart from a recommendation for foam dressings to reduce shear, with a strength of evidence summary at 'level B' (11).

Pressure ulcer prevention is expensive, ranging from $0.6 \%$ of all health care expenditure in Hungary (12) to $1 \%$ in the Netherlands (13) and as high as 4\% (£1.4-£2.1 billion annually) in the UK (14). The costs of pressure ulcers are reported to be much higher in the USA with recent

\section{Key Messages}

- dressings as an adjunct to pressure ulcer prevention

- five-layer soft silicone bordered dressings have been shown to reduce the incidence of pressure ulcers when combined with usual pressure ulcer prevention practices

- pressure ulcer prevention in critically ill patients should include a five-layer soft silicone bordered dressing on the sacrum to reduce pressure, shear and microclimate

- pressure ulcer prevention should begin in the Emergency Department for patients likely to be admitted for surgery or to critical care units

estimates of \$6-\$15 billion annually for all types of chronic wounds (including pressure ulcers) (15); however, in Japan 
the reported costs of pressure ulcer treatment are much lower ( $£ 18.4$ million annually) (16). Costs for prevention and treatment of pressure ulcers that occur in acute care facilities in the USA are not reimbursed; pressure ulcers have been deemed as 'medical errors' and when they occur no funds are reimbursed even if the ulcer was unavoidable.

The formulation of recommendations upon the use of wound dressings in pressure ulcer prevention was undertaken by a group of experts in the field from Australia, Portugal, the UK and the USA. The expert panel recognises that it does not represent the NPUAP, European Pressure Ulcer Advisory Panel (EPUAP) or the Pan Pacific Pressure Injury Alliance. The consensus group met on six occasions over 2011 and 2012 augmented by regular teleconference calls. Their preliminary work was presented at several international meetings of wound care providers for input.

The foundations for the consensus-based recommendations was a formal search for, and review of, published literature related to dressing use in pressure ulcer prevention. In May 2011, a literature search was conducted within the following electronic databases (PUBMED, MEDLINE, CINHAL, AMED and EMBASE) along with hand-searching of conference proceedings [European Wound Management Association (EWMA) and EPUAP] to identify literature published in languages familiar to the consensus group (English or Spanish) from 1998 to 2011 that was related to wound dressings used in pressure ulcer prevention. Within the search strategy the detailed search developed by the NPUAP-EPUAP to identify publications upon pressure ulcer prevention, and the separate NPUAP-EPUAP search strategy used in their pressure ulcer treatment guideline to identify wound dressings were combined to search for pressure ulcer prevention and the various types of wound dressing available to clinicians. This search had not been performed within the 2009 NPUAP EPUAP guidelines ${ }^{1}$ and represents a new contribution to the evidence base that underpins pressure ulcer prevention. The initial literature search identified 595 possibly relevant publications; these were reviewed by a single investigator and excluded if (a) there was no abstract available or (b) were review articles or (c) the abstract identified that the study was either unrelated to the use of wound dressings (e.g. considered the use of under-pads and padding in prevention) or described the use of wound dressings in wound treatment.

This initial assessment reduced the number of potential studies from 595 to 35 potential publications. The full and reduced publication lists were made available to the consensus group to ensure potential papers had not been excluded incorrectly. Evidence tables were developed based upon the structure of the evidence tables developed during the 2009 NPUAP-EPUAP guideline development process. All evidence tables were reviewed by the consensus group with the agreement that ten potential studies should be excluded (not about pressure ulcer prevention or involved skin creams and gels rather than dressings). This review left the consensus group with 25 eligible studies. The review was updated in September 2012 with further 403 publications identified that had been published since the initial literature search.
Following the same process used in the first search, the 403 publications were reduced to 15 potential studies for inclusion with the consensus group excluding 12 of these with the final 3 eligible for inclusion in the review. This gave a total of 28 included publications which formed the basis for the review and consensus recommendations.

Twelve studies considered the clinical outcomes of wound dressing use on pressure ulcer prevention, 11 reported measurements of pressure, shear or friction where wound dressings were applied to the skin, the final five publications all in the Spanish language appeared to be duplicate publications or where an English language version also existed. In addition, one of the members of the consensus panel was conducting a randomised control trial (RCT) for a dressing during this time. The consensus panel members were able to read the final manuscript and the findings of that study are also included in the literature review for this article.

Statements were written in the style of existing guidelines along with the level of evidence (A, B and C), defined as:

- Level A: Derived from high quality RCT.

- Level B: Derived from other evidence such as welldesigned, non-randomised clinical trial, clinical cohort studies and case-control studies with non-biased selection of study participants and consistent findings.

- Level C: Derived from expert opinion and may include data from other sources.

1. Consider the use of a five-layer soft silicone bordered foam dressing to enhance, but not replace, pressure ulcer prevention strategies for the sacrum, buttock and heel $(\mathrm{SOE}=\mathrm{A})$.

Evidence: Literature used to inform this recommendation includes one prospective randomised controlled trial (1), three cohort studies $(2,4)$ and two case series $(5,6)$.

Research from ICU: A prospective open-label randomised control trial using a five-layer soft silicone bordered dressing on the sacrum and a three-layer non-bordered dressing on the heel (Mepilex Border Sacrum ${ }^{\circledR}$ and Mepilex Heel ${ }^{\circledR}$ by Mölnlycke Health Care, Gothenburg, Sweden) in 440 patients with traumatic injury or critical illness reported that there were significantly fewer patients with pressure ulcers in the dressing group (7 versus $27, P=0.002$ ) (1). There were significantly fewer sacral pressure ulcers compared with a control group (5 versus $20, P=0.001$ ) and fewer heel ulcers (5 versus 19, $P=0.002$ ). This well-designed and adequately powered study examined 440 trauma and critically ill patients. The dressing was applied in the Emergency Department and maintained throughout the stay in intensive care unit (ICU). The control group received usual and customary care. The reduction in pressure ulcers represented a $10 \%$ difference in incidence between the groups $(3.1 \%$ versus $13.1 \%)$ and a number needed to treat of ten patients to prevent one pressure ulcer. The time to injury survival analysis indicated that intervention group patients had a hazard ratio of $0.19(P=0.002)$ compared with control group patients.

Three clinical trials also studied the effect of preventive dressings in ICU patients. Brindle and Wegelin (2) followed up 93 surgical trauma ICU patients considered to be at risk 
of pressure ulcers and treated preventively with a five-layer soft silicone bordered dressing on the sacrum. Of the 41 patients provided with the wound dressing, none developed sacral pressure damage whereas 3 of 52 patients considered to be less vulnerable to pressure ulcer development experienced sacral pressure ulcers.

Chaiken conducted a clinical trial in 293 patients primarily admitted to the ICU with brain, cardiac, infectious or respiratory problems (4). Baseline pressure ulcer rates were $13.6 \%$ in the ICU prior to the application of a five-layer soft silicone bordered dressing. In the first month after the study, the pressure ulcer rate fell to $1.8 \%(N=5)$.

Walsh et al. used a five-layer soft silicone bordered dressing in 62 patients on their admission to the ICU. Three of the 62 patients developed a sacral pressure ulcer, noted on days 3, 12 and 24 after dressing placement. Two of these ulcers were diagnosed as deep tissue injury and one as a stage II. Continued use of the dressing along with implementation of the pressure ulcer guidelines decreased the hospital-acquired rates from $12.5 \%$ to $7 \%$ in 1 year (6).

Research from $O R$ and ICU: Brindle and Wegelin conducted a trial in 85 patients admitted for surgery with planned admission to the ICU. Five-layer soft silicone bordered dressings were applied to all patients prior to surgery and then removed from the control group upon admission to ICU. No OR-acquired pressure ulcers developed in either group. Four subjects in the control group $(N=35)$ developed deep tissue injury and category (stage) II pressure ulcers 6 days after the operation. One deep tissue injury developed in the treatment group; however, this ulcer did not evolve. The differences were not statistically significant (5).

Cherry et al. (pers. commun.) examined the change in pressure ulcer rates after implementing a combined effort with the ORs and the cardiovascular ICU. The OR staff placed gel overlays on the OR tables for high-risk patients and ensured that no prep solution pooled against the skin. The ICU staff applied a five-layer silicone foam bordered dressing (Mepilex ${ }^{\circledR}$ ) for the patients upon arrival to ICU and inspected the skin beneath the dressing at every shift. In the first quarter after the changes, pressure ulcer rate was $0 \%$ compared with a pre-intervention rate of $8-14 \%$. In the second quarter after the changes, the pressure ulcer rate was $1 \%$.

While the findings from the RCT (1) provide preliminary evidence and the other studies supplement the findings on the effectiveness of a five-layer soft silicone bordered dressing to prevent pressure ulcers, the expert panel fully supports continuing standard pressure ulcer prevention practices and considering dressings as an adjunct to practices. Pressure ulcer prevention strategies include turning and repositioning to reduce the duration of pressure, using therapeutic support surfaces to reduce the magnitude of pressure, keeping the head of the bed at or below $30^{\circ}$ elevation to reduce the risk of shear, keeping the skin clean and dry to control microclimate and providing nutrition and hydration to maintain tissue tolerance for pressure. No clinical studies could be located that compared the pressure ulcer outcomes between dressing types.
2. Before selecting a dressing, consider the current status of the skin and the ease of dressing removal in order to prevent mechanical stripping $(\mathrm{SOE}=\mathrm{B})$.

Evidence: Skin injury can result from repeated removal of strongly adhesive dressings $(17,18)$. If skin is torn, easily bruised or fragile use a dressing such as soft silicone which is recognised to prevent skin damage (18). For patients with fragile skin, use of a retention bandage to hold the dressing securely in place is recommended.

3. Apply the dressing to dry intact skin. Do not use emollients or other barriers because they will prevent adhesion of the dressing to the skin $(\mathrm{SOE}=\mathrm{C})$.

Evidence: Several products are used on the skin to reduce the risk of incontinence-associated dermatitis. These products can reduce the adhesive properties of a dressing applied to the same skin and therefore the skin should be dry before application of a dressing. Dressings for prevention of pressure ulcers should not be used as containment products for incontinence.

4. Choose a dressing(s) that exceeds the area of tissue at risk on the sacrum, buttocks or heel to be protected from pressure and shear $(\mathrm{SOE}=\mathrm{C})$.

Evidence: When shear forces are applied to the skin, for example, when the head of the bed is elevated, the patient's skin, underlying tissue and the blood vessels are pulled and distorted in a wide area. Therefore, when using a dressing to 'absorb' the shear forces, it must be larger than the body area at risk for shear. If the dressing is smaller, the shear force is transmitted onto the skin and underlying soft tissues. The effect of shear can be seen in dressings; when the dressing is found to be wrinkled, the change in appearance of the dressing is due to shear being applied to the dressing and not the skin. So, in essence, the dressing did its job by absorbing the shear forces. It is not advisable to apply topical products or tape to secure the dressing in these instances. Excessive adhesion will damage the skin (19).

5. Inspect the skin beneath the dressing on a regular basis in accordance with standards of care and/or institutional policy $(\mathrm{SOE}=\mathrm{C})$.

Evidence: Guidelines on pressure ulcer prevention state that skin over body areas at high risk for pressure injury must be inspected often to detect early signs of pressure damage. When dressing for pressure ulcer prevention is used that cover the high-risk body areas, the skin must still be inspected. Therefore, the ability of the dressing to be removed without injury to the skin in order to inspect the skin needs is an important component within this recommendation.

6. Dressings should be changed in accordance with institutional policy and manufacturers recommendations or as clinically indicated $(\mathrm{SOE}=\mathrm{C})$.

Evidence: Dressings that become soiled or damaged may need to be changed more quickly than institutional policy or manufacturers' recommendations. 
7. Consider discontinuation of the dressing as the patient's risk for pressure ulcer development decreases per clinical assessment $(\mathrm{SOE}=\mathrm{C})$.

Evidence: As the patient's risk for pressure ulcers decreases, commonly seen once the patient is fully awake and aware following surgery, ambulating and/or moving purposefully in bed, preventive dressings may not be needed. The patient's overall risk must be considered; if the patient is only ambulating for a few minutes a day and relatively immobile once bedbound, continued use of the dressing should be considered. (See additional recommendations for heel ulcer prevention.)

8. Consider placement of five-layer soft silicone bordered foam dressing to the buttocks and sacrum prior to prolonged procedures or anticipated events when the patient cannot move or be moved from the supine position $(\mathrm{SOE}=\mathrm{B})$.

Evidence: A laboratory study created standardised pressure on seven dressings and analysed the area of pressure deflection created by the dressing (20). The data reported that the deflection of pressure through the dressing was greatest in the five-layer soft silicone dressing (Mepilex ${ }^{\circledR}$ ); therefore the dressing resulted in the lowest pressure applied to the skin. The test model used for the study was not human tissue; therefore the extent of pressure deflection into deeper tissue (e.g. muscle) is unknown. Literature also used to inform this recommendation has been presented above, however, because the duration of pressure and the position of the patient was not studied, these studies were not considered as primary evidence. These studies examined the outcomes from the use of a five-layer soft silicone bordered foam dressing during admission through the Emergency Department (ED) or surgical cases in which patients were admitted to the ICU. A study comparing pressure ulcer outcomes during surgery among 201 patients with and without a polyurethane film dressing reported reduced ulceration for 24 hours after surgery; however, duration of the operation was most predictive of ulceration (19). Studies specifically looking at the effect of dressings during interventional radiology transport by gurney or backboard could not be found. Immobile supine patients experience pressure on the buttocks and sacrum.

9. Consider placement of soft silicone dressings onto the buttocks and sacrum when the head of the bed must be continuously elevated $(\mathrm{SOE}=\mathrm{B})$.

Evidence: This recommendation was primarily supported by laboratory research comparing shear force onto various sacral shaped dressings. The laboratory study created standardised shear on seven dressings and analysed the deformation of the dressing and the artificial skin of the model in the laboratory (20). The data reported that the five-layer soft silicone bordered foam dressing (Mepilex ${ }^{\circledR}$ ) had the greatest ability to disperse the shear forces. The test model used for the study was not human tissue; therefore the extent of shear dispersement into deeper tissue (e.g. muscle) is unknown. Literature used to inform this recommendation has been presented above, however, because the elevation of the head of the bed was not reported, these studies were not considered as primary evidence. These studies examined the outcomes from the use of a five-layer soft silicone bordered foam dressing on patients in the ICU. It is presumed that the head of the bed was elevated in many of these patients while on ventilators.

10. Consider placement of multi-layer soft silicone foam dressings on the heels prior to prolonged procedures or anticipated events when the patient's leg(s) cannot move or be moved from the supine position $(\mathrm{SOE}=\mathrm{A})$.

Evidence: A prospective open-label randomised control trial using a three-layer soft silicone dressing (Mepilex Heel ${ }^{\circledR}$ dressings) in 440 patients with traumatic injury or critical illness reported that there were significantly fewer patients with heel ulcers (5 versus 19, $P=0.002$ ). The dressing was applied in the Emergency Department and maintained throughout the stay in ICU. The anatomy and physiology of the heel make the tissue very vulnerable to pressure. When pressure is increased in high-risk patients, such as those with peripheral arterial disease and neuropathy, the risk of ulceration and failure to heal is greatly increased. Patients at risk of increased pressure on the heel are those who cannot move their legs, such as patients who are anesthetised, paralysed or have undergone orthopaedic injury or surgery. These patients require additional precautions to prevent heel ulceration. Elevation of the heel from the surface of the bed, carts, OR table, etc is an important first step. However, there are some patients who cannot move the heel free from the bed, such as those with orthopaedic injuries. Laboratory testing of the multi-layer soft silicone dressing showed an ability to deflect pressure away from the point of impact into the dressing.

11. Consider placement of multi-layer soft silicone foam dressings to the heels for patients at risk of shear injury $(\mathrm{SOE}=\mathrm{B})$.

Evidence: This recommendation is based on the study presented above. However, because clinical situations that lead to shear injury of the heel were not described, the findings were not considered as primary data. The anatomy and physiology of the heel make the tissue vulnerable to friction and shear. While pressure causes compression of the tissues, shear forces occur between the layers of the tissues, and tend to tear and separate them. In some cases, this results in blister formation and breakdown of the fibres that tether the layers of fat and collagen together. The reduction of shear force was tested in the laboratory and the multi-layer soft silicone dressing showed an ability to deflect shear forces away from the point of impact into the dressing. Shear forces are best prevented with layers that move along with the shear forces.

\section{Skin microclimate}

No clinical studies were reported addressing the effect of a dressing on skin microclimate. Microclimate is being recognised again as an aetiology of pressure ulcer formation in that moist and warm skin has less tolerance for pressure (21). Laboratory data on the ability of dressings to absorb 
and trap moisture as well as allow moisture to escape were reviewed for this project (20).

\section{Conclusion}

Evidence on the effectiveness of five-layer soft silicone bordered dressing (Mepilex ${ }^{\circledR}$ ) supports its use as a component of sacral pressure ulcer prevention in patients at high risk of pressure ulcers, those in the ED, ICU and OR. A welldesigned and adequately powered RCT examined 440 trauma and critically ill patients and reported statistically significantly fewer pressure ulcers on the sacrum and heels in those treated with the dressing. Other studies and laboratory findings bolster this conclusion.

\section{Areas for further research}

There is a need for further research in the use of dressings for the prevention of pressure ulcers given the current strength of published evidence. Specifically, there is a need for large multi-site randomised controlled trials investigating the effectiveness of different types of dressing at different anatomical sites; these studies should investigate a broad range of patient populations, from critical care to medical-surgical populations as well as the aged care sector. Furthermore, we need to better explain the potential protective mechanisms involved in the use of dressings in pressure ulcer prevention at both cellular and biomechanical levels. This future research will be important to guide the development of specific 'preventive' dressings that may differ in their construction and mechanical properties from current wound dressings that have been used for pressure ulcer prevention (21).

\section{Acknowledgement}

The work of this panel of experts was supported by Mölnlycke Health Care, Gothenburg, Sweden.

\section{References}

1. Santamaria N, Gerdtz M, Sage S, McCann J, Freeman A, Vassilou T, DeVincentis S, Wei A, Manias E, Liu W, Knott J. A randomised controlled trial of the effectiveness of soft silicone foam multi-layer dressings in the prevention of sacral and heel pressure ulcers in trauma and critically ill patients: the border trial. Int Wound $J$ 2013; doi: 10.1111/iwj.12101.

2. Brindle CT, Wegelin JA. Prophylactic dressing application to reduce pressure ulcer formation in cardiac surgery patients. $J$ Wound Ostomy Continence Nurs 2012;39:133-42.

3. Chaiken N. Reduction of sacral pressure ulcers in the Intensive Care Unit using a silicone border foam dressing. J Wound Ostomy Continence Nurs 2012;39:143-5.
4. Brindle CT, Wegelin JA. Prophylactic dressing application to reduce pressure ulcer formation in cardiac surgery patients. $J$ Wound Ostomy Continence Nurs 2012;39:133-42.

5. Walsh NS, Blanck AW, Smith L, Cross M, Andersson L, Polito C. Use of a sacral silicone border foam dressing as one component of a pressure ulcer prevention program in an intensive care unit setting. J Wound Ostomy Continence Nurs 2012;39:146-9.

6. Panel for the Prediction and Prevention of Pressure Ulcers in Adults. Pressure Ulcers in Adults: Prediction and Prevention, 1992. Clinical Practice Guideline, No 3. Rockville, MD. U.S. Department of Health and Human Services. Public Health Service, Agency for Health Care Policy and Research. AHCPR Publication No 92-0047.

7. Pieper B, National Pressure Ulcer Advisory Panel (NPUAP), editors. Pressure ulcers: prevalence, incidence and implications for the future. Washington: NPUAP, 2012.

8. Cuddigan J. Critical care. In: Pressure ulcers: prevalence, incidence and implications for the future. Washington: NPUAP, 2013:47-56.

9. Ganos D, Siddiqui A. Pressure ulcers in the operating room. In: Pressure ulcers: prevalence, incidence and implications for the future. Washington: NPUAP, 2013:57-63.

10. National Pressure Ulcer Advisory Panel, European Pressure Ulcer Advisory Panel. Prevention and treatment of pressure ulcers: clinical practice guideline. Washington: National Pressure Ulcer Advisory Panel, 2009.

11. Gulacsi L. Hungarian Health Care in Transition. Studies on the improvement of the effectiveness of health care in Hungary by implementing quality assurance. PhD Thesis, University of Amsterdam 2001.

12. Severens JL, Habraken JM, Duivenvoorden S, Frederiks CM. The cost of illness of pressure ulcers in the Netherlands. Adv Skin Wound Care 2002;15:72-7.

13. Bennett GC, Dealey C, Posnett J. The cost of pressure ulcers in the UK. Age Ageing 2004;33:230-5.

14. Markova A, Mostow EN. US skin disease assessment: ulcer and wound care. Dermatol Clin 2012;30:107-11.

15. Sanada H, Nakagami G, Mizokami Y, Minami Y, Yamamoto A, Oe M, Kaitani T, Iizaka S. Evaluating the effect of the new incentive system for high-risk pressure ulcer patients on wound healing and cost-effectiveness: a cohort study. Int J Nurs Stud 2009;47:279-86.

16. Dykes PJ. The effect of adhesive dressing edges on cutaneous irritancy and skin barrier function. $J$ Wound Care 2007;16:97-100.

17. Waring M, Bielfeldt S, Mätzold K, Wilhelm KP, Butcher M. An evaluation of the skin stripping of wound dressing adhesives. $J$ Wound Care 2011;20: 412, 414,416-22.

18. Imanishi K, Morita K, Matsuoka M, Hayashi H, Furukawa S, Terashita F, Moriya E, Kanesaki U, Kinukawa N, Nose Y, Moroi Y, Urabe K, Furue M. Prevention of postoperative pressure ulcers by a polyurethane film patch. $J$ Dermatol 2006;33:236-7.

19. Call E, Pedersen J, Bill B, Black J, Alves P, Brindle CT, Dealey C, Santamaria N, Clark M. Enhancing pressure ulcer prevention using wound dressings: what are the modes of action? Int Wound J 2013; doi: 10.1111/iwj.12123.

20. Gefen A. How do microclimate factors affect the risk for superficial pressure ulcers: a mathematical modeling study. $J$ Tissue Viability 2011;20:81-8.

21. Clark M, Clarke M. Global evidence based practice recommendations for the use of wound dressings to augment pressure ulcer prevention protocols. Int Wound J 2013. In press. 